\title{
Circulating endothelial cells demonstrate an attenuation of endothelial damage by minimizing the extracorporeal circulation
}

Christian A. Skrabal, MD, ${ }^{a}$ Yeong H. Choi, MD, ${ }^{a}$ Alexander Kaminski, MD, ${ }^{a}$ Michael Steiner, MD, ${ }^{\mathrm{b}}$ Guenther Kundt, PhD, ${ }^{\mathrm{c}}$ Gustav Steinhoff, MD, PhD, and Andreas Liebold, MD, $\mathrm{PhD}^{\mathrm{a}}$

From the Department of Cardiac Surgery, ${ }^{\mathrm{a}}$ Institute of Clinical Chemistry and Laboratory Medicine, ${ }^{\mathrm{b}}$ and Institute of Medical Informatics and Biometry, ${ }^{\mathrm{c}}$ University of Rostock, Rostock, Germany.

Dr. Liebold received financial support from Maquet Cardiopulmonary Inc. Maquet Cardiopulmonary Inc had no involvement in the study design or interpretation of results.

Received for publication Dec 22, 2005; accepted for publication March 13, 2006.

Address for reprints: Christian A. Skrabal, MD, Department of Cardiac Surgery, University of Rostock, Schillingallee 35, D-18057 Rostock, Germany (E-mail: cskrabal@ fastmail.fm).

J Thorac Cardiovasc Surg 2006;132:291-6

$0022-5223 / \$ 32.00$

Copyright (C) 2006 by The American Association for Thoracic Surgery

doi:10.1016/j.jtcvs.2006.03.017
Objective: Detachment of endothelial cells may represent serious injury of the endothelium after cardiopulmonary bypass. We investigated whether the extent of endothelial injury is related to the type of cardiopulmonary bypass system used (conventional or minimized) and determined circulating endothelial cells as well as von Willebrand factor and soluble thrombomodulin.

Methods: Twenty patients scheduled for elective coronary bypass grafting were randomly assigned to either the minimal extracorporeal circulation system or the standard cardiopulmonary bypass. Ten healthy volunteers served as controls. Circulating endothelial cells per milliliter of full blood were perioperatively determined by immunomagnetic cell separation technique. Endothelial plasma markers were measured by enzyme-linked immunosorbent assay.

Results: Preoperative circulating endothelial cell numbers did not differ between the experimental groups, but were significantly higher than in the healthy controls (18.6 \pm 5.6 vs $7.2 \pm 3.8, P<.001)$. At 6 hours, circulating endothelial cell numbers increased significantly compared with baseline in both experimental groups and peaked at 12 hours after cardiopulmonary bypass initiation, each time with significantly lower values in the minimal extracorporeal circulation group (6 hours: $44.0 \pm 9.9$ vs $29.6 \pm 9.8, P=.007 ; 12$ hours: $48.1 \pm 6.8$ vs $31.8 \pm 7.1, P<.001)$. Likewise, von Willebrand factor and soluble thrombomodulin postoperatively increased in both groups with a tendency toward lower levels in the minimal extracorporeal circulation group. Although circulating endothelial cells gradually declined, continually with lower numbers in the minimal extracorporeal circulation group, the endothelial plasma markers remained elevated during observation time.

Conclusions: Circulating endothelial cells represent a novel marker of the intrinsic endothelial damage caused by cardiopulmonary bypass. Its analysis facilitates the evaluation of cardiopulmonary bypass modifications as the minimal extracorporeal circulation system could be proven to be less injurious to endothelium and myocardium.

$\mathrm{S}$ ince its first successful clinical use in the early 1950s, cardiopulmonary bypass (CPB) has undergone a variety of technical improvements. Nonetheless, there are recent trends in minimizing or completely avoiding the current $\mathrm{CPB}$, mainly to reduce the blood-foreign surface contact. According to the Society of Thoracic Surgeons National Database, serious complications develop in 20\% of low-risk patients after cardiac surgery. ${ }^{1}$ More than 20 years ago, Kirklin and colleagues $^{2}$ reported an activation of the complement system after CPB. It is common knowledge that CBP triggers a global defense reaction of the whole body, usually denoted as systemic inflammatory response syndrome. ${ }^{3,4}$ An option to attenuate the deleterious $\mathrm{CPB}$-related effects might be the strict reduction of foreign 
surfaces, avoidance of blood-air contact, and modification of the pump itself. The advantages of such a minimized system have been shown in clinical studies by our group and others. ${ }^{5,6}$ The minimal extracorporeal circulation (MECC) circuit used is a closed, fully heparin-coated, and preconnected extracorporeal circulation system consisting of a diffusion membrane oxygenator and a centrifugal pump but foregoing a reservoir for pericardial suction blood.

The contact activation of blood cells with artificial surfaces and air, the operative trauma itself, ischemia/reperfusion injury, hemodilution, and endotoxemia caused by intestinal hypoperfusion are the predominant triggers of complement activation, alteration of the cytokine steady-state, alteration of coagulation and fibrinolysis, activation of immune-competent cells, and endothelial damage. ${ }^{7}$ So far, assessment of endothelial function has been accomplished by analysis of specific plasma markers such as von Willebrand factor (vWf), soluble thrombomodulin (sTM), soluble E-selectin, tissue plasminogen activator, or soluble endothelial cell protein $\mathrm{C}$ receptor. More recently, a novel method for assessing vascular integrity was established: the determination of circulating endothelial cells (CECs) immunomagnetically separated from the peripheral blood. Under physiologic conditions, CECs occur in the blood of healthy individuals in the range of 5 cells $/ \mathrm{mL}$, whereas elevated numbers are found among those with sickle cell anemia, rickettsial, and cytomegalovirus infections, Behçet disease, various forms of vasculitis, and type 2 diabetes mellitus. ${ }^{8-10}$ Mutin and colleagues ${ }^{11}$ detected elevated CECs in patients with acute myocardial infarction and unstable angina. CECs have also been shown to correlate with disease status and response to treatment in patients with renal transplant rejection or acute myeloid leukemia. ${ }^{12,13}$

In this pilot study, we investigated the endothelial activation in patients with cardiovascular disease before, during, and after cardiac surgery. The endothelial disturbance was sought to delineate by enumeration of CECs and analysis of vWf and sTM. By randomly using the MECC system or standard $\mathrm{CPB}$, we evaluated the endothelial damage induced by each system.

\section{Patients and Methods}

The study was approved by the local ethical board. Twenty nondiabetic patients scheduled for elective coronary artery bypass graft surgery were prospectively randomized into 2 groups of 10 patients each according to the type of CPB used. Exclusion criteria were ejection fraction less than $25 \%$, age more than 80 years, emergency operation, redo or combined cardiac surgery, severe renal dysfunction with or without requiring dialysis, and hepatic disorder. The randomization plan (20 patients in 2 sub-blocks) was generated by an open-access web-based tool (http://www.tufts.edu/ gdallal/PLAN.HTM). For standard $\mathrm{CPB}$, a polypropylene, microporous $(0.2 \mu \mathrm{m})$, hollow-fiber oxygenator (Jostra Quadrox, Maquet Cardiopulmonary AG,
Hirrlingen, Germany) with cardiotomy suction reservoir was used with a roller pump (Jostra HL 20, Maquet Cardiopulmonary AG). The MECC system introduces a new polymethylpenten hollow-fiber oxygenator with a plasmatight diffusion membrane (Jostra Quadrox D, Maquet Cardiopulmonary AG) and a centrifugal pump (Jostra RotaFlow, Maquet Cardiopulmonary AG), whereas fundamental components are reduced to minimal elements as arterial and venous lines or completely omitted as the cardiotomy reservoir. Both systems including the oxygenators were heparin coated and ensured a nonpulsatile flow of 2.0 to $2.5 \mathrm{~L} \cdot \mathrm{min} \cdot \mathrm{m}^{2}$ body surface area. Ten healthy volunteers served as controls ( 7 males, 3 females) to evaluate the preoperative endothelial activation status of patients with cardiovascular disease.

\section{Anesthesia}

Anesthesia was induced with $1 \mu \mathrm{g} / \mathrm{kg}$ sufentanil, $0.2 \mathrm{mg} / \mathrm{kg}$ etomidate, $0.04 \mathrm{mg} / \mathrm{kg}$ midazolam, and $0.15 \mathrm{mg} / \mathrm{kg}$ cisatracurium. Anesthesia was maintained with sufentanil, totaling $1 \mu \mathrm{g} / \mathrm{kg} / \mathrm{h}$ and midazolam $0.1 \mathrm{mg} / \mathrm{kg} / \mathrm{h}$; supplemental sevoflurane was given until $\mathrm{CPB}$. No volatile drugs were added during $\mathrm{CPB}$.

\section{Operation}

Approximately $300 \mathrm{IU}$ heparin per kilogram of total body weight was administered for systemic anticoagulation to aim at a target activated coagulation time of 350 to 400 seconds. The arterial cannula was placed in the ascending aorta, and the venous 2-stage cannula was placed in the right atrium. Lines were connected with diligence to avoid gaseous bubbles. Myocardial protection was achieved by antegrade administration of Calafiore blood cardioplegia. Mild hypothermia $\left(31^{\circ} \mathrm{C}-32.5^{\circ} \mathrm{C}\right)$ was instituted immediately after $\mathrm{CPB}$ start, measured by an esophageal temperature probe. Low-dose aprotinin $\left(1 \times 10^{6} \mathrm{IU}\right)$ was administrated in the CPB priming solution. In addition, the anesthesiologist applied $1 \times 10^{6}$ IU aprotinin per hour of CPB. The aorta was crossclamped while the distal anastomoses were completed. The proximal vein anastomoses were established with partial occlusion of the ascending aorta while the patient was rewarmed. Blood from the surgical site was collected in the cardiotomy reservoir in the standard CPB group or in a cell-saving device in the MECC group. Thereby, cell saving was processed at a certain blood volume $(>0.5 \mathrm{~L})$, and salvaged blood was retransfused only if necessary. After CPB, protamine ( $1 \mathrm{mg} / 100$ units of total heparin) was administered to antagonize heparin.

\section{Samples and Endothelial Markers}

Analysis was performed preoperatively, 30 minutes, 6 hours, 12 hours, 24 hours, and 48 hours after CPB initiation. Blood was collected into citrate tubes and centrifuged at $3345 \mathrm{~g}$ for 15 minutes (Heraeus Multifuge $1 \mathrm{~S}-\mathrm{R}$, Osterode, Germany), and supernatant was frozen at $-70^{\circ} \mathrm{C}$ for batch analysis. vWf antigen $(\mathrm{Ag})$ was measured by immunoturbidimetric determination using the Dade Behring vWf:Ag test kit (Dade Behring Marburg GmbH, Marburg, Germany). For determination of sTM concentration a commercial solid-phase sandwich enzyme-linked immunosorbent assay kit was used (human sCD141 ELISA kit, Diaclone Research, Besançon, France). 


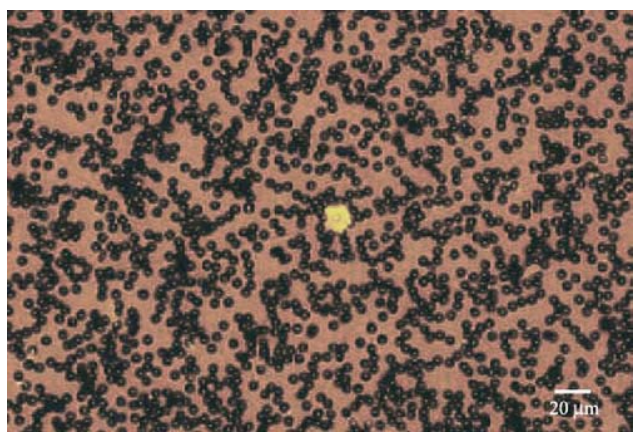

Figure 1. Representative microphotography of a rhodaminelabeled CEC caught by several magnetobeads. Fluorescent microscopy, $20 \times$ magnification.

\section{Circulating Endothelial Cell Analysis}

Blood samples for CEC quantification were taken last in EDTA tubes to avoid contamination by endothelial cells from the vessel wall. CEC quantification was done immediately or if unavoidable no longer than 8 hours after collection and storage at $4^{\circ} \mathrm{C}$. All laboratory work was performed in blinded fashion with respect to the identity of the samples.

To isolate CECs, we used M-450 Dynabeads, 4.5- $\mu \mathrm{m}$ diameter polystyrene beads coated with rat anti-mouse immunoglobulin-G1 (Dynal, Hamburg, Germany) coupled with murine anti-human CD146 antibody (Biocytex, Marseilles, France). One milliliter of blood was diluted with $1 \mathrm{~mL}$ of phosphate-buffered saline (PBS) containing $0.1 \%$ bovine serum albumin (BSA). Twenty microliters of FcR blocking agent (Miltenyi, Bergisch-Gladbach, Germany) were added to prevent nonspecific leukocyte binding and incubated with $100 \mu \mathrm{L}$ anti-CD146-coated Dynabeads $\left(8 \times 10^{6}\right.$ beads $/ 10 \mu \mathrm{L}$ antiCD 146) for 60 minutes while gently agitating in a head-over-head mixer. Cells bound to anti-CD146-coupled beads were separated from blood in a magnet (Dynal MPC), washed 4 times with PBS-BSA, and incubated in $100 \mu \mathrm{L}$ of rhodamine-labeled UlexEuropaeus-Agglutinin-1 solution (1:10 dilution, Linaris Wertheim, Germany) for 1 hour on an orbital shaker in darkness. Cells were washed, resuspended in $100 \mu \mathrm{L}$ PBS-BSA, and counted in a Nageotte chamber (Brand, Wertheim, Germany) with a fluorescent microscope (Leica DMLB, Leica Microsystems GmbH, Wetzlar, Germany) equipped with an excitation filter N2 (BP 546/12). In accordance with others, CECs were identified as well-delineated round or oval rhodamine-labeled cells with a size of 10 to $40 \mu \mathrm{m}$ and with more than 4 beads attached. ${ }^{14}$ Enumeration of CECs was done 2 times each sample, in case of deviation, and the average value was taken. Figure 1 shows a CEC caught by several magnetobeads.

\section{Statistical Analysis}

All data were stored and analyzed using the SPSS statistical package 12.0 (SPSS Inc. Chicago, Ill). Descriptive statistics were computed for variables of interest. The computed statistics included mean and standard deviations. Because of the small number of patients and controls and the low power resulting in a failure to falsify a null hypothesis, statistical tests regarding normal distri-
TABLE 1. Patients' demographics

\begin{tabular}{lccc}
\hline Criteria & Standard CPB & MECC & $P$ value \\
\hline Number (n) & 10 & 10 & \\
Age $(\mathrm{y})$ & $65.2 \pm 9.6$ & $67.5 \pm 10.3$ & .61 \\
Male/female & $8 / 2$ & $7 / 3$ & .27 \\
Preoperative EF & $0.50 \pm 0.07$ & $0.46 \pm 0.15$ & .55 \\
Previous MI $(<30 \mathrm{~d})$ & $4(0)$ & $5(1)$ & .65 \\
CPB time (min) & $102.6 \pm 24.0$ & $90.2 \pm 35.4$ & .27 \\
Grafts & $3.80 \pm 0.79$ & $3.50 \pm 0.85$ & .42 \\
ICU stay (d) & $1.1 \pm 0.31$ & $1.1 \pm 0.31$ & .99 \\
\hline
\end{tabular}

MECC, Minimal extracorporeal circulation; $C P B$, cardiopulmonary bypass; $E F$, ejection fraction; $M I$, myocardial infarction; $I C U$, intensive care unit.

bution of variables were not carried out. Consequently, nonparametric tests were used. For the preoperative moment, values were available for all 3 groups; therefore, differences were analyzed with the Kruskal-Wallis test and, if necessary, in pairs between the groups with the Mann-Whitney $U$ test including Bonferroni correction, which means an alpha level of 0.05/3 $=0.017$. After CPB initiation, values were available only in the 2 experimental groups, and comparisons were made with the Mann-Whitney $U$ test each time (alpha level $=0.05 / 5=0.01$ ). Within-group comparisons between all moments were made with the Friedman test. If the hypothesis of equality of all 6 moments was rejected, comparisons between the preoperative moment and the other 5 postoperative times were made by using Wilcoxon tests with Bonferroni correction (alpha level $=0.05 / 5=0.01)$. Generally, all $P$ values resulted from 2-sided tests and without Bonferroni correction a $P$ value of less than .05 was considered to indicate statistical significance.

\section{Results}

Patients' perioperative demographics are shown in Table 1. There was no statistical difference between the experimental groups regarding age, gender ratio, preoperative ejection fraction, CPB duration, number of grafts, and intensive care unit stay. Four patients in the standard CPB group and 5 patients in the MECC group had a history of myocardial infarction. One patient in the MECC group had a recent myocardial infarction within the last 30 days (19 days). The postoperative course was uneventful in all patients. Only 1 patient in the standard CPB group required homologous blood transfusion on postoperative day 2. Retransfusion of cell-saver blood was not required in any of the patients in the MECC group.

\section{Circulating Endothelial Cells}

Preoperative CEC numbers (cells per milliliter of blood) did not differ between the experimental groups (standard CPB: $17.8 \pm 3.9$, MECC: $19.3 \pm 7.1 ; P=.971)$ but were significantly higher than in the healthy controls $(7.2 \pm 3.8$; $P<.001$ for both of the 2 comparisons) (Figure 2). During $\mathrm{CPB}, \mathrm{CECs}$ demonstrated only a slight elevation. At 6 hours, CECs increased significantly compared with baseline in both groups and peaked at 12 hours after CPB initiation, 


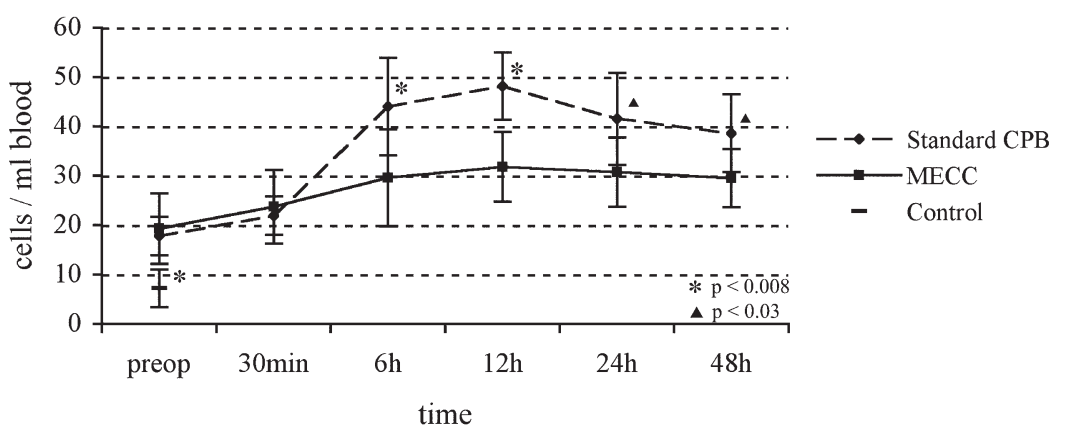

Figure 2. Course of circulating endothelial cells (CECs) in patients with cardiovascular disease before, during, and after surgery. CECs increased significantly in both groups post-CPB versus pre-CPB, each time with lower numbers in the MECC group versus standard CPB group. CEC enumeration in healthy controls indicated a higher endothelial baseline activity in patients with cardiovascular disease. CPB, Cardiopulmonary bypass; MECC, minimal extracorporeal circulation. each time with significantly lower values in the MECC group (6 hours: $44.0 \pm 9.9$ vs $29.6 \pm 9.8, P=.007$; 12 hours: $48.1 \pm 6.8$ vs $31.8 \pm 7.1, P<.001)$. At 24 and 48 hours, CECs gradually decreased again with lower numbers in the MECC group (24 hours: $41.5 \pm 9.3$ vs $30.7 \pm 7.0, P=.011$; 48 hours: $38.6 \pm 7.9$ vs $29.5 \pm 5.9, P=.023)$. Friedman tests documented significant changes in CECs in both experimental groups $(P<.001)$. At any time, postoperative values were significantly higher than initial values (Wilcoxon test: $P=.005$ for each test).

\section{Von Willebrand Factor Antigen}

Preoperative vWf:Ag (international units/deciliter) did not differ between the experimental groups (standard CPB: $143.7 \pm 48.9$, MECC: $126.4 \pm 33.7$; Mann-Whitney $U$ test: $P=.436)$, but its concentration strongly tended to higher values in both groups of patients with cardiovascular disease compared with the healthy controls (99.5 \pm 22.1 , standard CPB: Mann-Whitney $U$ test: $P=.019$; MECC: Mann-Whitney $U$ test: $P=.075$ ) (Figure 3). After surgery, vWf:Ag increased in both experimental groups (Friedman test: $P<.001$, Wilcoxon test: $P=.005$ for each test). Even if the postoperative plasma concentrations of vWf:Ag tended to be lower in the MECC group (6 hours: $191.1 \pm$ 59.4 vs $230.0 \pm 83.0 ; 12$ hours: $215.3 \pm 65.5$ vs $267.3 \pm$ $68.0 ; 24$ hours: $242.9 \pm 64.9$ vs $298.8 \pm 76.2$; 48 hours: $311.1 \pm 76.4$ vs $359.8 \pm 56.4$ ), differences between the groups were not statistically significant (Mann-Whitney $U$ test: $P>.085$ for each test).

\section{Soluble Thrombomodulin}

Plasma concentration of sTM (nanograms/milliliter) was similar in preoperative cardiovascular patients and healthy controls (standard CPB $2.3 \pm 1.3$, MECC $1.8 \pm$ 0.9 , controls $1.7 \pm 0.8$; Kruskal-Wallis test: $P=.638$ ) (Figure 4). Friedman tests documented significant changes within both experimental groups $(P<.001)$. Whereas in the standard CPB group the postoperative sTM concentrations were significantly higher than preoperative concentrations at any time (Wilcoxon test: $P=$ .005 for each test), only the 48-hour increase was statistically significant (Wilcoxon test: $P=.005$ ) in the MECC group. Nonetheless, there was no difference between the groups at any postoperative time.

\section{Discussion}

To our knowledge, this is the first study investigating the CPB-related endothelial activation by evaluation of CECs. The particular feature of CECs is that they not only unveil endothelial damage but also correlate with the activity and degree of endothelial injury. ${ }^{15,16} \mathrm{CECs}$ in our healthy volunteers were in the range of 7 cells $/ \mathrm{mL}$, which meets current results of other groups. ${ }^{10,17}$ Mutin and colleagues ${ }^{11}$ observed elevated CECs in patients 18 to 24 hours after acute myocardial infarction, whereas patients with stable angina did not show such phenomena. However, we detected high CEC numbers preoperatively in our elective patients with stable angina. Assuming Mutin and colleagues used the same separation

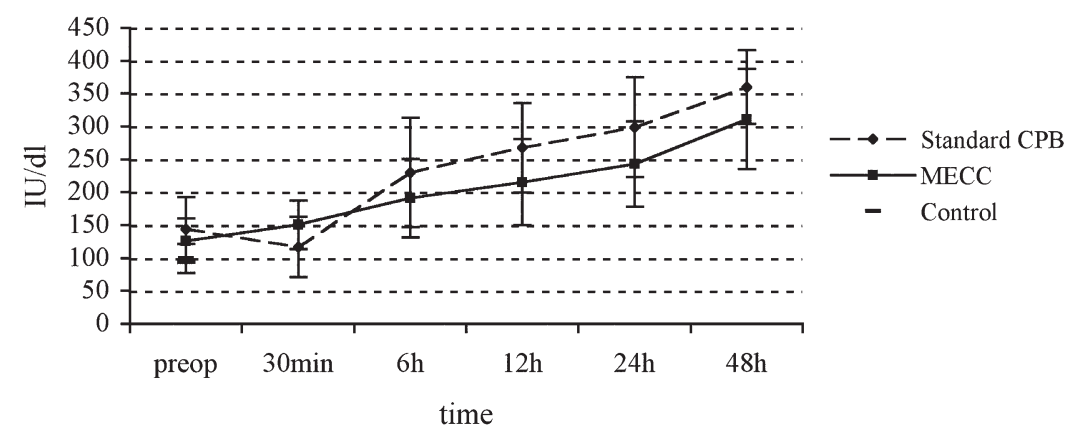

Figure 3. von Willebrand factor (vWf) plasma concentration increased significantly after CPB versus preoperative with a tendency toward lower values in the MECC group versus standard CPB group. vWf values of controls were lower than preoperative values of patients with cardiovascular disease. CPB, Cardiopulmonary bypass; MECC, minimal extracorporeal circulation. 


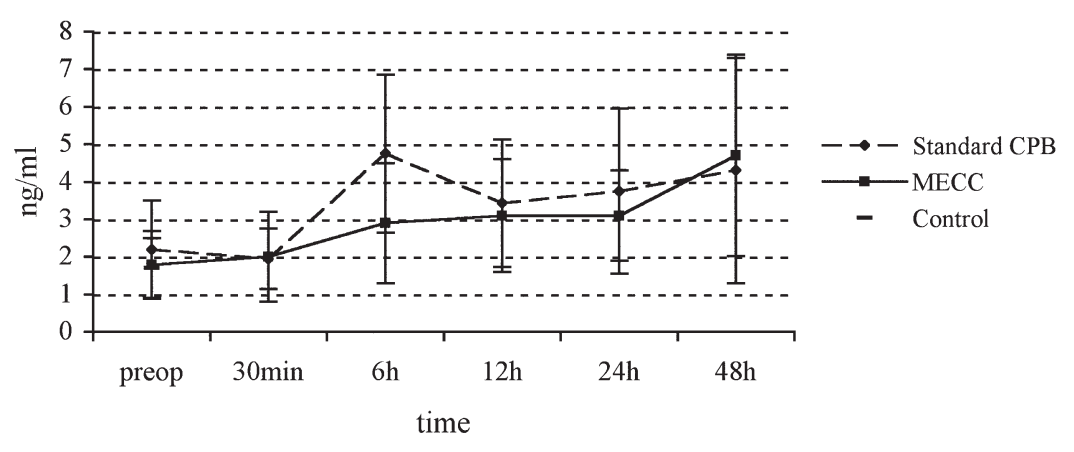

Figure 4. Soluble Thrombomodulin (sTm) increased significantly post-CPB versus pre-CPB. Baseline values of patients with cardiovascular disease did not differ from those of controls. CPB, Cardiopulmonary bypass; MECC, minimal extracorporeal circulation.

technique and criteria for endothelial cell identification, we conclude that there has to be a difference in the severity of patients' cardiovascular atherosclerosis. Particularly, if these results are compared with the results in our healthy volunteers, we have to presume a higher endothelial baseline activity in patients with cardiovascular disease that might be supported by likewise elevated vWf. sTM was not elevated in our patients with cardiovascular disease, at least not preoperatively. This closely resembles the results of Chong and colleagues, ${ }^{18}$ who observed a positive correlation between CECs and vWf but not between CECs and sTM.

The fact that CECs increase significantly after CPB might indicate an additional or new endothelial disturbance. Because CECs remained nearly unchanged in both setups during $\mathrm{CPB}$, we do not assume a mechanical trigger like direct vascular manipulation during cannulation or surgery. The delayed increase more likely indicates a mediated mechanism of endothelial detachment caused by direct neutrophil attack, cytokines, and proteases. ${ }^{10}$ In agreement with the theory that activated neutrophils interfere with endothelial cell contact, Scholz and colleagues ${ }^{19}$ investigated the modulation of endothelial junction molecules by neutrophils isolated from patients undergoing cardiac surgery before and during CPB. They observed an impairment of the endothelial integrity by relocation of the zonula adherens molecule $\beta$-catenin caused only by neutrophils isolated at the end of CPB. Due to these results, CPB triggers a time-dependent activation of neutrophils that dissolve the endothelial connectivity and induce detachment of endothelial cells. The detached endothelial cells are floating in the bloodstream and can be detected by sufficiently sensitive methods. CD146 is expressed almost exclusively on mature endothelial cells, the exception being some tumor cell lines. $^{20}$ The immunomagnetic isolation technique we used is a modified method used by Woywodt and colleagues. ${ }^{14}$ In addition, we determined endothelial plasma markers such as vWf and sTM, which are known to be related to endothelial damage and capable of predicting prognostic outcomes. ${ }^{18,21,22} \mathrm{CECs}$, vWf, and sTM were lowest preoperatively, remained low during CPB, and were elevated 6 hours after CPB initiation in both groups. While vWf and sTM further increased during observation time, CECs peaked at 12 hours and decreased further on.

One major aim of our study was the comparison of 2 different CPB setups. Although the endothelial markers responded similarly to $\mathrm{CPB}$ in both groups, the absolute values were lower or at least tended to be lower in the MECC group. In regard to the differences in the CPB setups, we assume that any use of different components might have an impact on inflammation-mediated endothelial function. The cardiotomy suction device increases the artificial surface area and introduces a blood-air interface, which both trigger a strong activation of cellular components. ${ }^{23,24}$ Retransfusion of pericardial suction blood reinforces the inflammatory response. ${ }^{25,26}$ Several studies revealed advantages of centrifugal over-roller pumps in regard to cellular activation, especially in the long term. ${ }^{27-29}$ Finally, the oxygenators, even if structurally almost identical, differ with regard to the hollow-fiber material and tightness. The membrane in the Quadrox D (MECC system) has no pores, thus preventing air from entering the circuit. Furthermore, the special oxygenator coating aims at decreasing the inherent pressure gradient resulting in less hemolysis and decreased release of inflammatory mediators. However, in our study we compared the CPB systems as whole units well knowing that the influence of each component requires further analysis.

One of the limitations of this study is the relatively small number of patients in both experimental groups and the inequality between study groups and controls. Enrolling more patients would have increased the power of the study. Additional CEC isolation from different sampling sites, for example, the coronary sinus or pulmonary veins, would have delivered new insights into the origin of CECs and the potential correlation to organ ischemia. Stepwise modification of CPB would have allowed statements about the importance of the particular components for endothelial damage. All this must be subjected to further studies. 


\section{Conclusion}

CECs represent a novel marker enabling the assessment of endothelial injury. In contrast to well-established plasma markers, CECs seem to be more accurate in detecting the intrinsic endothelial damage. Patients with cardiovascular disease not only have a higher endothelial baseline activity than the healthy population but also sustain additional damage contingent on the CPB system used. Our results indicate that the MECC system may be less injurious to endothelium than the standard CPB. As this was a pilot study, further investigations are needed to validate our observations.

\section{References}

1. Grover FL. The Society of Thoracic Surgeons National Database: current status and future directions. Ann Thorac Surg. 1999;68:367-73; discussion 374-6.

2. Kirklin JK, Westaby S, Blackstone EH, Kirklin JW, Chenoweth DE, Pacifico AD. Complement and the damaging effects of cardiopulmonary bypass. J Thorac Cardiovasc Surg. 1983;86:845-57.

3. Ascione R, Lloyd CT, Underwood MJ, Lotto AA, Pitsis AA, Angelini GD. Inflammatory response after coronary revascularization with or without cardiopulmonary bypass. Ann Thorac Surg. 2000;69:1198204.

4. Asimakopoulos G. Mechanisms of the systemic inflammatory response. Perfusion. 1999;14:269-77.

5. Fromes Y, Gaillard D, Ponzio O, Chauffert M, Gerhardt MF, Deleuze $\mathrm{P}$, et al. Reduction of the inflammatory response following coronary bypass grafting with total minimal extracorporeal circulation. Eur J Cardiothorac Surg. 2002;22:527-33.

6. Wiesenack C, Liebold A, Philipp A, Ritzka M, Koppenberg J, Birnbaum DE, et al. Four years' experience with a miniaturized extracorporeal circulation system and its influence on clinical outcome. Artif Organs. 2004;28:1082-8.

7. Paparella D, Yau TM, Young E. Cardiopulmonary bypass induced inflammation: pathophysiology and treatment. An update. Eur J Cardiothorac Surg. 2002;21:232-44.

8. McClung JA, Naseer N, Saleem M, Rossi GP, Weiss MB, Abraham NG, et al. Circulating endothelial cells are elevated in patients with type 2 diabetes mellitus independently of $\mathrm{HbA}(1) \mathrm{c}$. Diabetologia. 2005;48:345-50.

9. Haubitz M, Woywodt A. Circulating endothelial cells and vasculitis. Intern Med. 2004;43:660-7.

10. Dignat-George F, Sampol J. Circulating endothelial cells in vascular disorders: new insights into an old concept. Eur J Haematol. 2000;65: 215-20.

11. Mutin M, Canavy I, Blann A, Bory M, Sampol J, Dignat-George F. Direct evidence of endothelial injury in acute myocardial infarction and unstable angina by demonstration of circulating endothelial cells. Blood. 1999;93:2951-8.

12. Wierzbowska A, Robak T, Krawczynska A, Wrzesien-Kus A, Pluta A, Cebula B, et al. Circulating endothelial cells in patients with acute myeloid leukaemia. Eur J Haematol. 2005;75:492-7.

13. Woywodt A, Schroeder M, Gwinner W, Mengel M, Jaeger M, Schwarz A, et al. Elevated numbers of circulating endothelial cells in renal transplant recipients. Transplantation. 2003;76:1-4.
14. Woywodt A, Goldberg C, Scheer J, Regelsberger H, Haller H, Haubitz M. An improved assay for enumeration of circulating endothelial cells. Ann Hematol. 2004;83:491-4.

15. Woywodt A, Streiber F, de Groot K, Regelsberger H, Haller H, Haubitz M. Circulating endothelial cells as markers for ANCA-associated smallvessel vasculitis. Lancet. 2003;361:206-10.

16. Solovey A, Lin Y, Browne P, Choong S, Wayner E, Hebbel RP. Circulating activated endothelial cells in sickle cell anemia. $N$ Engl J Med. 1997;337:1584-90.

17. Woywodt A, Bahlmann FH, De Groot K, Haller H, Haubitz M. Circulating endothelial cells: life, death, detachment and repair of the endothelial cell layer. Nephrol Dial Transplant. 2002;17:1728-30.

18. Chong AY, Blann AD, Patel J, Freestone B, Hughes E, Lip GY. Endothelial dysfunction and damage in congestive heart failure: relation of flow-mediated dilation to circulating endothelial cells, plasma indexes of endothelial damage, and brain natriuretic peptide. Circulation. 2004;110:1794-8.

19. Scholz M, Nowak P, Blaheta R, Schuller A, Menon S, Cinatl J, et al. Relocalization of endothelial cell beta-catenin after coculture with activated neutrophils from patients undergoing cardiac surgery with cardiopulmonary bypass. J Invest Surg. 2004;17:143-9.

20. Bardin N, George F, Mutin M, Brisson C, Horschowski N, Frances V, et al. S-Endo 1, a pan-endothelial monoclonal antibody recognizing a novel human endothelial antigen. Tissue Antigens. 1996;48:531-9.

21. Salomaa V, Matei C, Aleksic N, Sansores-Garcia L, Folsom AR, Juneja $\mathrm{H}$, et al. Soluble thrombomodulin as a predictor of incident coronary heart disease and symptomless carotid artery atherosclerosis in the Atherosclerosis Risk in Communities (ARIC) Study: a casecohort study. Lancet. 1999;353:1729-34.

22. Conway DS, Pearce LA, Chin BS, Hart RG, Lip GY. Prognostic value of plasma von Willebrand factor and soluble P-selectin as indices of endothelial damage and platelet activation in 994 patients with nonvalvular atrial fibrillation. Circulation. 2003;107:3141-5.

23. Gong J, Larsson R, Ekdahl KN, Mollnes TE, Nilsson U, Nilsson B. Tubing loops as a model for cardiopulmonary bypass circuits: both the biomaterial and the blood-gas phase interfaces induce complement activation in an in vitro model. J Clin Immunol. 1996;16:222-9.

24. Gourlay T, Samartzis I, Taylor KM. The effect of haemodilution on blood-biomaterial contact-mediated CD11b expression on neutrophils: ex vivo studies. Perfusion. 2003;18:87-93.

25. Aldea GS, Soltow LO, Chandler WL, Triggs CM, Vocelka CR, Crockett GI, et al. Limitation of thrombin generation, platelet activation, and inflammation by elimination of cardiotomy suction in patients undergoing coronary artery bypass grafting treated with heparin-bonded circuits. J Thorac Cardiovasc Surg. 2002;123:742-55.

26. Westerberg M, Bengtsson A, Jeppsson A. Coronary surgery without cardiotomy suction and autotransfusion reduces the postoperative systemic inflammatory response. Ann Thorac Surg. 2004;78:54-9.

27. Andersen KS, Nygreen EL, Grong K, Leirvaag B, Holmsen H. Comparison of the centrifugal and roller pump in elective coronary artery bypass surgery-a prospective, randomized study with special emphasis upon platelet activation. Scand Cardiovasc J. 2003;37:356-62.

28. Linneweber J, Chow TW, Kawamura M, Moake JL, Nose Y. In vitro comparison of blood pump induced platelet microaggregates between a centrifugal and roller pump during cardiopulmonary bypass. Int $J$ Artif Organs. 2002;25:549-55.

29. Parolari A, Alamanni F, Naliato M, Spirito R, Franze V, Pompilio G, et al. Adult cardiac surgery outcomes: role of the pump type. Eur J Cardiothorac Surg. 2000;18:575-82. 